\title{
Rapid Observation with an Atmospheric Scanning Electron Microscope
}

\author{
H. Nishiyama, ${ }^{*}$ M. Suga, ${ }^{*}$ M. Koizumi, ${ }^{*}$ K. Ogawa, ${ }^{* *}$ Y. Konyuuba, ${ }^{*}$ D. Guarrera, ${ }^{* * *}$ \\ and C. Sato ${ }^{* * * *}$ \\ * JEOL Ltd., 1-2 Musashino 3-chome, Akishima, Tokyo 196-8558, Japan \\ ** JEOL Technics Ltd., 6-38 Musashino 2-chome, Akishima, Tokyo 196-0021, Japan \\ *** JEOL USA Inc., 11 Dearborn Road, Peabody, MA 01960, USA \\ ${ }^{* * * *}$ National Institute of Industrial Science and Technology (AIST), Tsukuba, 305-8566, Japan
}

A new atmospheric scanning electron microscope (ASEM): ClairScope is able to directly observe a sample in liquid or gas in the atmosphere [1-3]. In this instrument, the SEM has been redesigned to be placed upside down: its gun is set at the bottom whereas an ASEM dish with a sealed SiN window is placed onto a sample stage at the top as shown in Fig. 1. A thin SiN film is only $100 \mathrm{~nm}$ in thickness which is transparent to electrons and can withstand atmospheric pressure. For SEM imaging, the electron beam is scanned upward on a sample in the ASEM dish through the $\mathrm{SiN}$ window and the backscattered electrons from the sample through the $\mathrm{SiN}$ window are captured by a detector situated below.

In this presentation, as one of especially useful applications are reported a few examples of rapid observation of samples: several kinds of cheeses and metal pastes etc. without any sample pretreatment.

As the first example, ASEM images of two kinds of cheeses are shown in Figs. $2 \mathrm{a}$ and $2 \mathrm{~b}$. Protein clusters and voids are observed and their distributions are clearly different. The ASEM quickly and easily provides this kind of information. These kinds of finer features may affect flavor and texture of food, especially fermented food like cheese, so that the characterization of these features with the ASEM can be used for the quality control of these products.

As the second example, ASEM images of a silver paste are shown in Figs. $2 \mathrm{c}$ and $2 \mathrm{~d}$. It is to be noted that the image of Fig. $2 d$ is taken at a magnification of 20,000. Metal pastes for wiring on electronic devices are constantly being inspected to keep their quality, which is controlled by the shape and dispersion of particles and the type and amount of contaminants. Cryo-SEM and TEM are now used for the inspection. But they require skilled operators. Furthermore, they require much time for the data acquisition and analysis as well as the sample preparation. In the case of ASEM, the sample is just placed on the ASEM dish without pretreatment. Once the dish is put on the sample stage, observed images at high magnification can be taken in less than 10 minutes: about 2 minutes for column evacuation and less than a minute for focusing. Focus adjustment is easy even for operators who are not familiar with a 
SEM, because a working distance is set to be almost constant.

These two examples strongly indicate a high potential of the ASEM for monitoring of industrial processes and inspection of products.

\section{References}

[1] H. Nishiyama et al., J Struct Biol 169, 438-449 (2010).

[2] T. Murai et al., J Biol Chem 286, 1999-2007 (2010).

[3] http://www.jeol.com/PRODUCTS/ElectronOptics/ScanningElectronMicroscopesSEM /HighVacuumLowVaccum/JASM6200ClairScope/tabid/714/Default.aspx
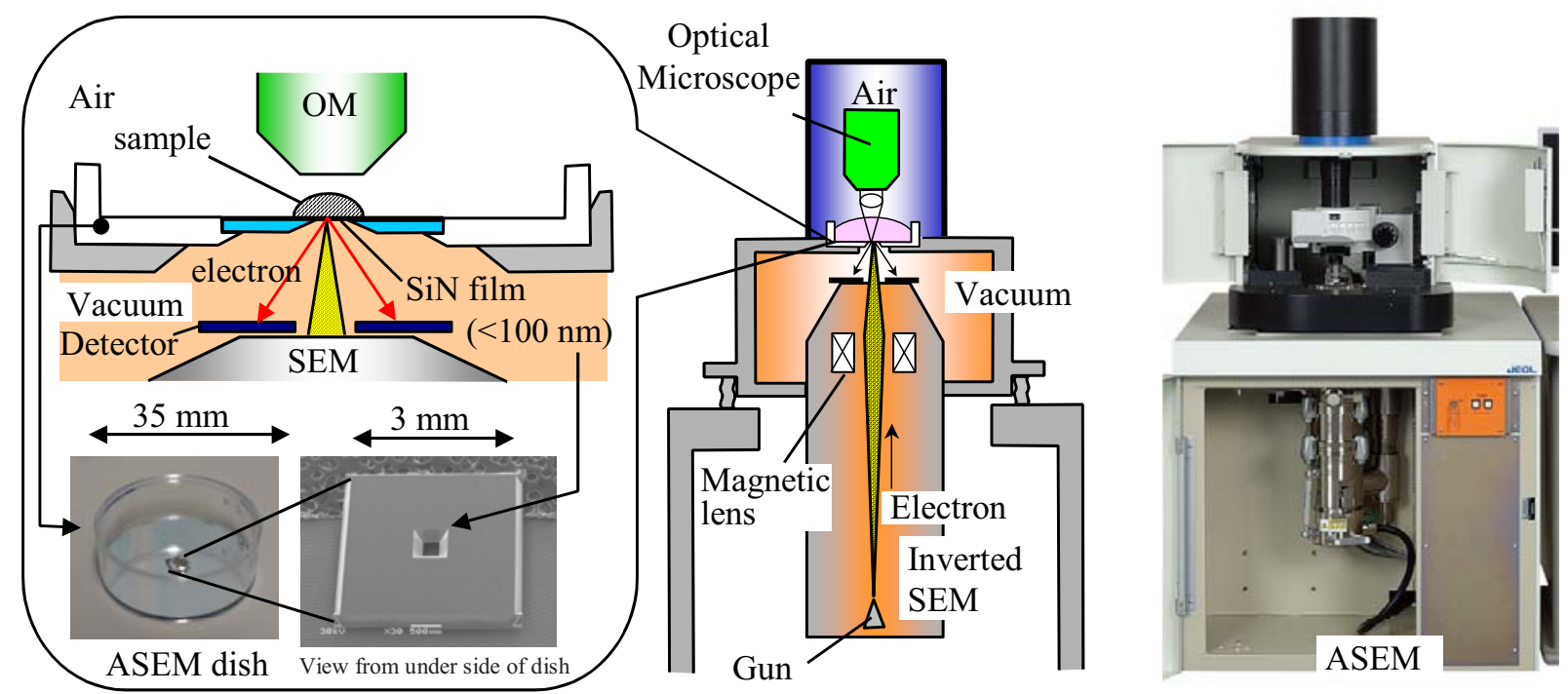

Fig. 1. Configuration of the ASEM: ClairScope. An optical microscope is installed opposite an inverted SEM with a ASEM dish in between. The ASEM dish has a sealed SiN window at the bottom. A film covering the SiN window, which is thin enough to transmit an electron beam, can withstand atmospheric pressure. The electron beam is scanned on a sample through the SiN window and the backscattered electrons from the sample are detected for high resolution of SEM images.
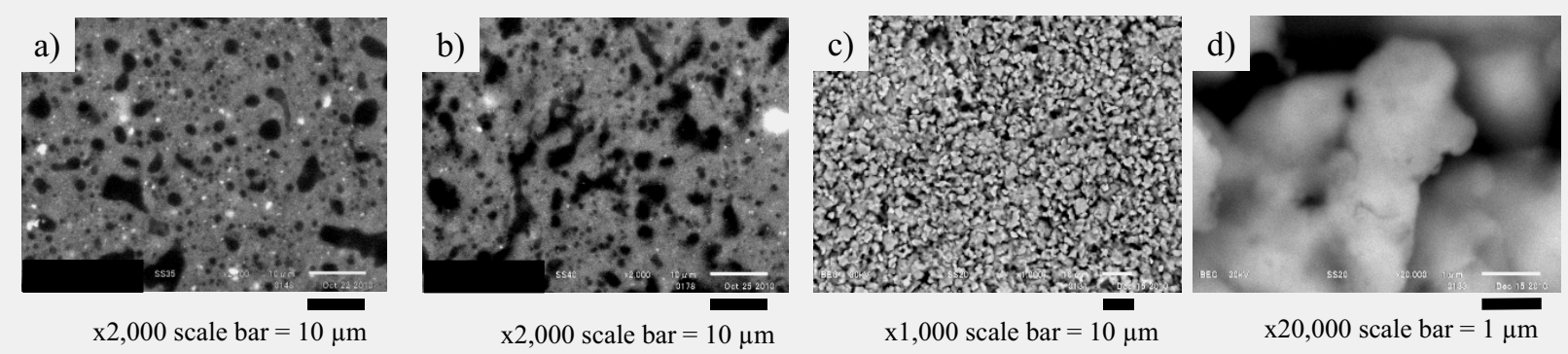

Fig. 2. ASEM images: a) cheese A, b) cheese B, c) a silver paste, d) magnified image of c. 